\title{
Escape time formulation of state estimation and stabilization with quantized intermittent communication
}

\author{
Chun-Chia Huang ${ }^{\text {a }}$, Robert R. Bitmead ${ }^{\text {a }}$

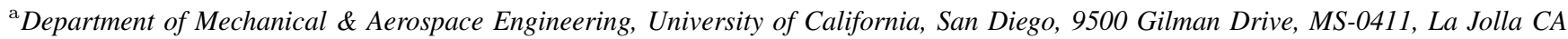 \\ 92093-0411, USA.
}

\begin{abstract}
The problems of state estimation and feedback stabilization of a linear system including a communications channel are quantified as escape or survival times, which yield stochastic processes describing the time of first exit of the state estimate error or of the system output from a specific domain. The complications introduced by communications - intermittency, channel noise, quantization, etc - are evaluated using a Markov stopping time formulation. This is compared to and contrasted with earlier analyses which considered the behavior of Kalman filters with intermittent data based on moments and conditional moments, and the evaluation of the minimal number of bits required for mean square stabilization. The main result shows the escape time is characterized by a Markov chain which is amenable to explicit analysis through the calculation of its cumulative distribution function. This is examined in the linear Gaussian and quantized linear Gaussian cases and then used to develop an approach to bitrate assignment in such communications-based control systems.
\end{abstract}

\section{Introduction}

We study the problems of state estimation and output feedback stabilization of a time-invariant linear system including a single communications link.

$$
\begin{aligned}
x_{k+1} & =A x_{k}+B u_{k}+w_{k}, \\
y_{k} & =C x_{k}+v_{k}, \\
z_{k} & =\gamma_{k} \mathcal{Q}_{d}\left(y_{k}\right) .
\end{aligned}
$$

Here, as usual, $x_{k}, u_{k}, y_{k}, w_{k}, v_{k}$ are the system state, input, output, process noise and measurement noise signals of dimensions $n, p, m, n, m$ respectively and $[A, B, C]$ are the system matrices of conformable dimensions. The signal $z_{k}$ is the received measurement from the system and is modeled in (3) as the product of a scalar random variable, $\gamma_{k}$, taking values 0 or 1 and a quantized version of the system output $y_{k}$. As in $[20,19]$, the intermittency of the communication channel is modeled by the $\left\{\gamma_{k}\right\}$ sequence. The quantization function $\mathcal{Q}_{d}(\cdot)$ is a subtractive dithered quantizer with finite bitrate in each channel of input $y_{k}$ and will be further explicated shortly.

\section{Definition 1 (Escape time) Given a closed domain $\mathcal{D} \subset$}

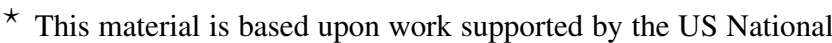
Science Foundation under Grant No. 1102384. An early version of this paper was presented at the 2013 European Control Conference [7].

Email addresses: chhuang@ucsd.edu (Chun-Chia Huang), rbitmead@ucsd. edu (Robert R. Bitmead).
}

$\mathbb{R}^{d}$ and a stochastic process $\left\{\xi_{k}: k=1, \ldots\right\}$ on $\mathbb{R}^{d}$, the escape time is defined to be

$$
\tau_{e}= \begin{cases}\arg \min _{k} \xi_{k} \notin \mathcal{D}, & \text { or } \\ \infty, & \text { if } \xi_{k} \in \mathcal{D} \quad \forall k\end{cases}
$$

Sometimes the escape time is called the 'first exit time,' 'stopping time,' 'hitting time' or 'residence time.' We shall be concerned with the escape time for the state process, $x_{k}$, state prediction error process, $\tilde{x}_{k \mid k-1}$, or the output process, $y_{k}$, of (1-2) when the control input is causally computed.

Figure 1 shows two simulations of the Kalman state prediction error $\tilde{x}_{k \mid k-1}$ for a scalar system with a magnitude bound placed at 60 and differing rates, $P_{\gamma}$, of packet arrival. In the left graph, the expected value of the conditional error covariance - the focus of [20] - is finite, while in the right it is not. Note the similarities between the figures except for the time scales. Escape time would correspond to the first achievement of the bound 60. Our interest is in characterizing this escape time cumulative distribution function.

Clearly the escape time is a random variable provided the infinite value has zero probability. We have the following result applicable in the linear Gaussian case and independent of the system matrices $[A, B, C]$.

Lemma 1 For the linear system (1), with noise process $\left\{w_{k}\right\}$ Gaussian, white, possessing full-rank covariance and 

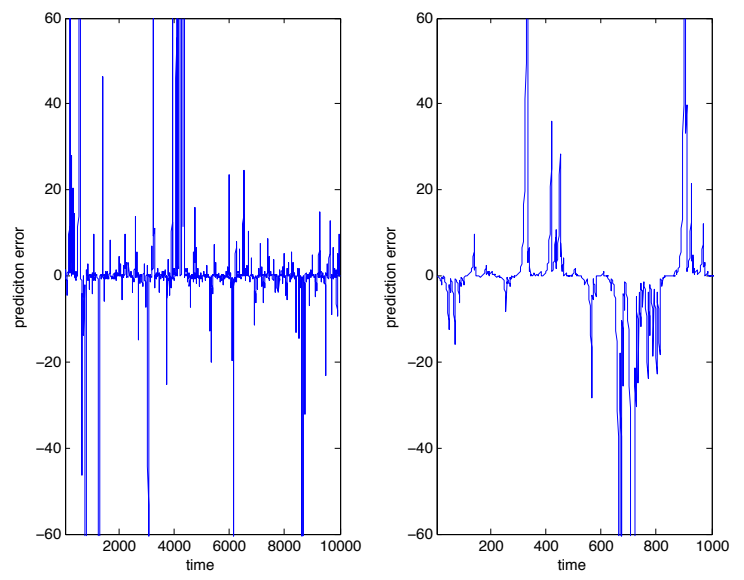

Fig. 1. Simulation of Kalman predictor error with $A=1.2, C=1$, $Q=0.005, R=0.001, P_{\gamma}=0.15$ (left) and 0.1 (right), and bound level 60 .

independent from $x_{1}$, with control $u_{k}$ causally computed, and for $\mathcal{D}$ compact, the escape time of $x_{k}$ is almost surely finite.

The proof is in the Appendix. The import of Lemma 1 is that it ensures that, in the linear Gaussian case or equivalent problems able to be transformed to linear Gaussian, using say Girsanov's Theorem, the finite escape of the state and/or output from any compact domain is ensured. The analysis of such processes then ought to concentrate on the description of the escape time rather than attempting to establish almost sure confinement to a compact set or characterize moment properties. This hearkens back to the escape time or residence time analysis of, say, $[10,24,32,12,5]$. These earlier treatments focus on stable continuous-time systems with small stochastic perturbations and use the Theory of Large Deviations to develop escape time characterizations as the noise power tends to zero. Our approach will maintain discrete time and deal with both stable and unstable systems with non-infinitesimal perturbations. This will not draw on Large Deviations Theory other than for comparison.

Our treatment of (1-3) endeavors to blend two distinct trains of research. The first is associated with the behavior of state estimators for such systems as treated in, say, $[20,19,9,16]$ with or without control being applied. Since the system is linear and if the applied control is known, the controlled state behavior is derivable from the estimator. The second class of problems, characterized by results such as $[23,22,17,31]$ concentrates on the stabilization aspects of the feedback control. The distinction between the two sets of problems in the literature rests with the description of the communications channel and the adaptation of quantization. The work in $[15,14]$ studies the earlier work in a more general case and yields necessary conditions for stabilization, recovering results of some previous works in the two approaches. In the estimator problem, the communication is taken to be intermittent - that is, the stochastic process $\left\{\gamma_{k}\right\}$ operates in a persistent fashion to cause arbitrarily long outages of communications - but the communication is not limited in bitrate (there is no quantizer) and full state reconstruction occurs with any successful communication packet. In earlier work on the stabilization problem, the emphasis is on the quantizer and its associated bitrate limit and the channel is assumed not intermittent, i.e. $\gamma_{k}=1$ for all $k$, with a deterministic maximal delay and possible additive channel noise. The approach adopted in this paper is to permit both intermittency and limited bitrate, since the Markov model describing escape time applies to both. We also pose a different set of questions dealing with escape time, which we regard as being more apropos for these problems. These focus not on limiting behaviors or mean-square stabilization but on characterizing the cumulative probability distribution function (cdf) of the escape time of the system state, output or state estimate error, since in general there is no almost sure bound on these, as stated in Lemma 1.

Before launching into the analysis, it is pertinent to examine some practical sources of estimation and control problems associated with systems described by (1-3), since the presence of a single communications link rules out teleoperationstyled feedback control problems. Utility management of a geographically distributed system, such as a power grid or radar network, where the sensors, but not the actuators, are remotely placed and linked back to base by communications networks, is the clearest application of state estimation operating with communications limits. The study of sensor fusion and its sibling area of sensor scheduling $[3,4]$ has a long history in these arenas. Schweppe [18] was a pioneer in the application of such methods in power system state estimation using data of variable reliability. More generally, the study of missing data has been longstanding in statistics [11] and in array beamforming [21] with studies of estimation in high noise going back to Wiener [26], whose work was connected with the origins of radar.

Closing the loop on a system to achieve stabilization using communicated data would appear to be a more recent problem. Interestingly, Wong and Brockett study first the state estimation problem [29] and then the feedback stabilization problem [30] for systems with reliable but bandlimited communications. This extends earlier results due to Williamson [27, 28] and Delchamps [2] on finite-wordlength effects on estimation and control in deterministic contexts. Sensor scheduling is also a feedback control or decision problem with a solution achievable via dynamic programming. The unifying aspect of these earlier analyses of the estimation and the control problems is that the covariance function of the state estimate error or of the state itself is the target of the analysis. Thus, mean-square stabilization is the objective in Nair and Evans [17].

For communications problems with intermittence, the conditional covariance, $\Sigma_{k+1 \mid k}$, of the state prediction error is a random process adapted to the $\left\{\gamma_{k}\right\}$ sequence. Recent works have been concerned with the distributional and moment properties of this $\left\{\Sigma_{k+1 \mid k}\right\}$ process. Thus: Sinopoli et al. [20] consider the convergence of the expectation $\mathrm{E}\left[\Sigma_{k+1 \mid k}\right]$ 
as $k \rightarrow \infty$; [8] models the packet dropping channel as the a two state Markov chain and arrives at the stabilization condition as in [20] for the scalar case; Shi, Epstein and Murray [19] quantify the probability $\operatorname{Pr}\left(\Sigma_{k+1 \mid k}>G\right)$ for given matrix $G$; [9] analyzes conditions for the weak (in distribution) convergence of $\Sigma_{k+1 \mid k}$; and, [16] treats the tail distribution properties of this covariance. (In this latter reference 'tail' refers to the distribution on the tail $\sigma$-algebra as $k \rightarrow \infty$ and not the tail probabilities in the sense of $\Sigma_{k+1 \mid k}$ taking on large values for any specific value of $k$.)

The problem formulation is presented in Section 2 and the concepts of escape and survival times are introduced for the estimation and stabilization problems. Section 3 develops the central Markov chain description of the escape time problem in a general context before examining this for Gaussian and quantized Gaussian linear systems. These results pertain when the packet arrival sequence, $\left\{\gamma_{k}\right\}$, is known. Section 4 presents numerical examples to demonstrate: the method, heuristics of escape time, and the quality of approximations. The analysis is then extended to explore approaches to quantization and retransmission in the output stabilization escape time. The Appendix follows the conclusion and contains the proofs.

Some of the material of this paper was presented in preliminary form in [7]. This present paper completes these results for the estimation problem by including theoretical, quantization and bit-rate assignment analyses together with computational approaches to bounding the escape time cdfs. This paper also extends the results of [7] to encompass output feedback stabilization of the underlying control system. Lastly, this paper corrects an embarrassing error in Theorem 3 dealing with the computation of the escape cdf. This error was pointed out by the reviewers of this paper, for which we are very grateful and for which we provide some comment.

\section{Problem formulation}

\subsection{Assumptions}

We commence with the communications-linked control system described by (1-3) and make the following assumptions.

Assumption $11.1 x_{1}$, the initial state, is Gaussian with mean $\bar{x}_{1}$, and covariance $\Sigma_{1 \mid 0}$.

$1.2 u_{k}=-K \hat{x}_{k \mid k}$ constant linear state filter feedback is applied.

$1.3 w_{k}$ and $v_{k}$ are independent, white, zero-mean Gaussian processes independent from $x_{1}$ and with covariance matrices $Q$ and $R$, which we take to be positive definite.

$1.4\left\{\gamma_{k}\right\}$ is a Bernoulli random process independent from $x_{1},\left\{w_{k}\right\}$, and $\left\{v_{k}\right\}$ and taking values 0 or 1 to describe the non-arrival or arrival of a data packet, respectively.
$1.5 \mathcal{Q}_{d}$ is a subtractive, dithered, b-bit-per-channel, midrise, symmetric, linear quantizer with saturation values $\pm \zeta$. The subtractive dither signal is white, triangularly distributed, $\operatorname{tr}\left(-\zeta / 2^{b-1}, \zeta / 2^{b-1}\right)$, independent from all other signals on the transmitter side, and known exactly to both transmitter and receiver.

A number of extensions are possible at the expense of clarity of development. (i) Time-variation can be included into the noise covariance matrices and into the feedback control gain. Stationarity of the problem does permit the calculation of expectations which converge over time as in [20]. However given Lemma 1, our focus is not on limiting behaviors but explicitly on transient properties, where time-variation is readily accommodated. (ii) In Assumption 1.4 we assume the process $\left\{\gamma_{k}\right\}$ is Bernoulli, as in [20]. It might equally well be taken as Markov as in [9]. (iii) Extension to nonlinear forms of quantization is straightforward. Extension to adaptive quantizers, while it is at the heart of Nair's and Evans' [17] demonstration of the minimal feedback bitrate required for mean-square stabilization, comes at the cost of communications and is not part of our analysis.

\subsection{Incorporating quantization}

Subtractive dithered $b$-bit quantizer, $\mathcal{Q}_{d}(\cdot)$, is depicted in Figure 2. It includes the fixed quantizer $\mathfrak{Q}$ and subtractive dither signal, $d_{k}$.

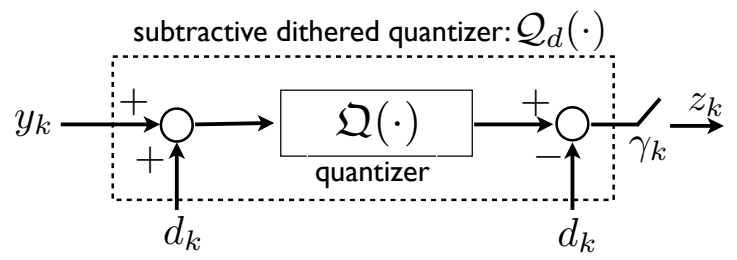

Fig. 2. Subtractive dithered quantization with intermittent gain $\gamma_{k}$.

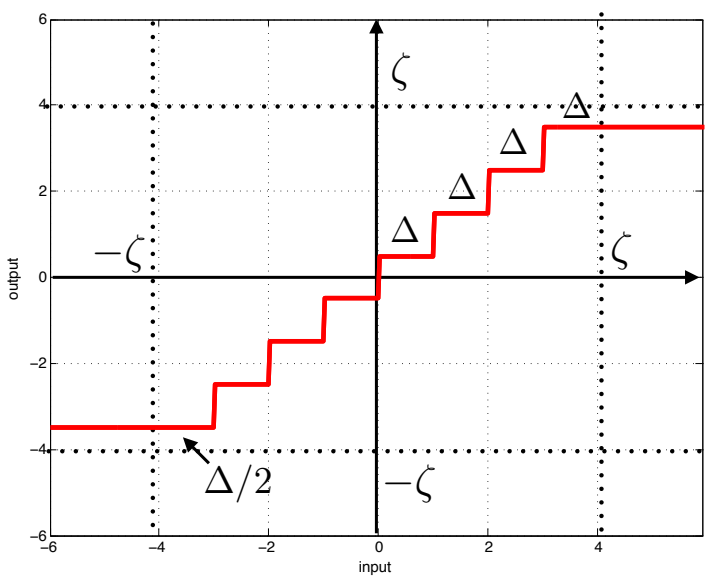

Fig. 3. The linear quantizer $\mathfrak{Q}$

The fixed quantizer function $\mathfrak{Q}(\cdot)$ is a mid-rise, symmetric, linear quantizer with $2^{b}$ levels within the range $[-\zeta, \zeta]$. 
Figure 3 shows a 3 -bit quantizer $\mathfrak{Q}$, the basic unit of the quantizer is $\Delta=\zeta / 2^{b-1}$. We note that subtractive dithered quantization requires careful handling of the wordlength of the subtraction operation, since $d_{k}$ is recorded at a higher bitrate than $b$ bits, see [25].

Theorem 1 Under the conditions of Assumption 1.5, and provided $y_{k} \in[-\zeta, \zeta]$, the quantization noise,

$$
n_{k}=\mathcal{Q}_{d}\left[y_{k}\right]-y_{k}=\left[\mathfrak{Q}\left(y_{k}+d_{k}\right)-d_{k}\right]-y_{k},
$$

is white, independent from $\left\{y_{k}\right\}$, and uniformly distributed $U\left(-\zeta / 2^{b}, \zeta / 2^{b}\right)$.

Quantization noise $n_{k}$ is a deterministic function of $y_{k}$ and $d_{k}$. The independence of $n_{k}$ and $y_{k}$ and the uniform distribution of $n_{k}$ from the theorem statement follow directly from Theorem QTSD of [25]. The whiteness of $\left\{n_{k}\right\}$ itself and its independence from $\left\{y_{j}: j \neq k\right\}$ follow from the whiteness of $\left\{d_{k}\right\}$ and its independence from $\left\{y_{k}\right\}$.

The effective measurement noise in $z_{k}$ is the sum of two independent terms, one Gaussian (measurement noise) and the other uniform (quantization noise).

$$
v_{\mathrm{eff}, k}=v_{k}+n_{k}
$$

In our subsequent analysis, we show how to compute the escape times with any measurement noise process but for clarity specialize to either a strictly Gaussian measurement noise or a Gaussian-plus-uniform measurement noise. With independent $m$-vectors $v_{k} \sim N\left(0_{m}, R\right)$ and $n_{k} \sim U^{m}\left(-\zeta / 2^{b}, \zeta / 2^{b}\right)$ the pdf of $v_{k}+n_{k}$ is given by

$$
\begin{aligned}
f_{v+n}(z)=\frac{2^{b-1}}{\zeta}\{\Phi & {\left[R^{-1 / 2}\left(z+\frac{\zeta}{2^{b}}\right)\right] } \\
& \left.-\Phi\left[R^{-1 / 2}\left(z-\frac{\zeta}{2^{b}}\right)\right]\right\}
\end{aligned}
$$

where $\Phi(\cdot)$ is the multivariate standard normal cdf of appropriate dimension. This is the convolution of the Gaussian pdf of $v_{k}$ with the uniform pdf of $n_{k}$.

When a quantizer is present and for $y_{k} \in[-\zeta, \zeta]$, the effective measurement noise covariance for $z_{k}$ increases from $R$ with perfect reconstruction of $y_{k}$ to

$$
R_{\mathrm{eff}}=R+\frac{\zeta^{2}}{3 \times 2^{2 b}} I_{m}
$$

which is the measurement noise, $v_{k}$, covariance plus the quantizer noise, $n_{k}$, covariance since these two noises are additive in (4) and are independent. With quantization, this quantity replaces $R$ in the Kalman filter recursion, which relies solely on second-order statistics and yields the least mean squares linear unbiased state estimator. (See Section 3.2 of [1].)
Outside of the range $[-\zeta, \zeta]$ the quantization error becomes potentially unbounded and correlated. In our situation of escape time analysis with fixed quantization, once the outer levels are breached the state or state estimation error has escaped. Nair and Evans in [17] develop an ingenious approach to adaptive quantization amenable to mitigating the effects of saturation errors by expanding $\zeta$ faster than the state can escape and, further, to achieving this within the allocated bitrate. In Section 5, strategies are studied to mitigate the effects of intermittency through the allocation of a fraction of the $b$ bits in the communicated signal to the coarse retransmission of earlier values of $y_{k}$. These studies extend earlier studies of signal reconstruction with missing data [6] and can deal with strategies for bit assignment in both TCP/IP and UMDP settings depending on the transmitter's knowledge of the arrival of packets.

\subsection{Kalman state estimation with intermittent quantized observations}

The Kalman state estimation equations with intermittent quantized observations described by $\left\{\gamma_{k}\right\}$ and $\mathcal{Q}$, commencing from initial values $\hat{x}_{1 \mid 0}=\bar{x}_{1}$ and $\Sigma_{1 \mid 0}$, are as follows. These equations are identical to those of $[20,19]$ and subsequent works modulo the incorporation of quantization via $R_{\text {eff }}$ and $n_{k}$.

$$
\begin{aligned}
& \text { measurement update } \\
& \qquad \begin{array}{l}
L_{k}=\gamma_{k}\left[\Sigma_{k \mid k-1} C^{T}\left(C \Sigma_{k \mid k-1} C^{T}+R_{\mathrm{eff}}\right)^{-1}\right] \\
\hat{x}_{k \mid k}=\hat{x}_{k \mid k-1}+L_{k}\left(z_{k}-C \hat{x}_{k \mid k-1}\right) \\
\quad \Sigma_{k \mid k}=\Sigma_{k \mid k-1}-L_{k} C \Sigma_{k \mid k-1} \cdot \\
\text { time update } \\
\hat{x}_{k+1 \mid k}=A \hat{x}_{k \mid k}+B u_{k} \\
\Sigma_{k+1 \mid k}=A \Sigma_{k \mid k} A^{T}+Q \\
\text { filtered error } \\
\quad \tilde{x}_{k \mid k}=x_{k}-\hat{x}_{k \mid k} \\
\text { prediction error } \\
\tilde{x}_{k+1 \mid k}=x_{k+1}-\hat{x}_{k+1 \mid k} \\
\quad=\left(A-A L_{k} C\right) \tilde{x}_{k \mid k-1}+w_{k}-A L_{k}\left(v_{k}+n_{k}\right)
\end{array}
\end{aligned}
$$

Note the $\gamma_{k}$ appears explicitly solely in the Kalman gain $L_{k}$ and that, conditioned on $\left\{\gamma_{k}\right\}$, the state prediction error system (10) is linear, time-varying due to $\gamma_{k}$, and driven by not-necessarily-Gaussian white noise. The Kalman predictor error equation (10) is central to both the estimator and the output escape time formulations below. We note that, when $\gamma_{k}=0$, then $L_{k}=0$ and (10) will be unstable if $A$ has eigenvalues outside the unit circle and driven by $w_{k}$ alone. If $\gamma_{k}=0$ for all $k$ - a case where we refer to the escape time as the survival time - (10) is time-invariant, which simplifies the analysis. 


\subsection{Output feedback control with intermittent and quan- tized observations}

The output feedback control problem with intermittent and quantized observations differs from the estimator problem since it involves both the filter error, $\tilde{x}_{k \mid k}$, and the controlled plant state, $x_{k}$. It is described by the linear system:

$$
\begin{gathered}
{\left[\begin{array}{c}
x_{k+1} \\
\tilde{x}_{k+1 \mid k} \\
y_{k+1}
\end{array}\right]=\left[\begin{array}{ccc}
A-B K & B K\left(I-L_{k} C\right) & 0 \\
0 & A\left(I-L_{k} C\right) & 0 \\
C(A-B K) & C B K\left(I-L_{k} C\right) & 0
\end{array}\right]\left[\begin{array}{c}
x_{k} \\
\tilde{x}_{k \mid k-1} \\
y_{k}
\end{array}\right]} \\
+\left[\begin{array}{c}
-B K L_{k}\left(v_{k}+n_{k}\right)+w_{k} \\
-A L_{k}\left(v_{k}+n_{k}\right)+w_{k} \\
-C B K L_{k}\left(v_{k}+n_{k}\right)+C w_{k}+v_{k+1}
\end{array}\right],
\end{gathered}
$$

This system's state covariance matrix is shown as follows,

$$
\operatorname{cov}\left(\left[\begin{array}{c}
x_{k} \\
\tilde{x}_{k \mid k-1} \\
y_{k}
\end{array}\right]\right)=\left[\begin{array}{ccc}
P_{k} & \Sigma_{k \mid k-1} & P_{k} C^{T} \\
\Sigma_{k \mid k-1} & \Sigma_{k \mid k-1} & \Sigma_{k \mid k-1} C^{T} \\
C P_{k} & C \Sigma_{k \mid k-1} & C P_{k} C^{T}+R
\end{array}\right]
$$

The Kalman filter recursion for intermittent quantized data, (7-9), yields the controlled state covariance, $P_{k}$, recursion

$$
\begin{aligned}
P_{k+1}= & (A-B K) P_{k}(A-B K)^{T} \\
& +(A-B K) \Sigma_{k \mid k-1}\left(I-L_{k} C\right)^{T} K^{T} B^{T} \\
& +B K\left(I-L_{k} C\right) \Sigma_{k \mid k-1}(A-B K)^{T} \\
& +B K\left(I-L_{k} C\right) \Sigma_{k \mid k-1}\left(I-L_{k} C\right)^{T} K^{T} B^{T} \\
& +B K L_{k} R_{\mathrm{eff}} L_{k}^{T} K^{T} B^{T}+Q .
\end{aligned}
$$

The effect of increasing the measurement noise through quantization is two-fold; the error covariance, $\Sigma_{k+1 \mid k}$, increases and then, as a consequence, the controlled state covariance, $P_{k}$, also increases. Additionallly, $R_{\text {eff }}$ directly drives (12). During periods of packet loss, measurement noise and quantization noise do not affect the system directly, since $L_{k}=0$. At times of packet arrival however, the quantization noise effect in increasing the underlying state estimate covariance and controlled state covariance is evident.

\subsection{Escape times, conditional escape times and survival times}

We have established that for output signals, $y_{k}$, within the quantization range $[-\zeta, \zeta]$, both the estimator error and the output feedback controlled system are governed by linear systems driven by white, not-necessarily-Gaussian noises: $\{(7),(8),(9),(10)\}$ for the estimator; and additionally (11) for the controlled system. This will form the basis for our calculation of escape times.

We make the following definitions.

Definition 2 (Estimator escape time $\tau_{e}$ ) For given positive scalar bound $G$, we define the estimator escape time to be the escape time for the random sequence $\left\{\tilde{x}_{k+1 \mid k}\right\}$ starting from the initial condition $\tilde{x}_{1 \mid 0} \sim N\left(0, \Sigma_{1 \mid 0}\right)$ and with $\mathcal{D}=\left\{\left|\tilde{x}_{k+1 \mid k}\right|_{\infty} \leq G\right\}$. The estimator escape time cdf is the cdf of $\tau_{e}$ given these initial conditions.

Definition 3 (Output escape time $\tau_{o}$ ) Given the output quantizer magnitude upper bound, $\zeta$ from Assumption 1.5, and given initial state covariance matrix $P_{1}$ and state estimate error covariance matrix $\Sigma_{1 \mid 0}$, we define the output escape time to be the escape time from $\mathcal{D}=\left\{\left|y_{k}\right|_{\infty} \leq \zeta\right\}$ with initial condition satisfying $y_{1} \sim N\left(0, C P_{1} C^{T}+R_{\mathrm{eff}}\right)$.

The estimator and output escape times are random variables depending on: the bound $\zeta$, the initial covariances $\Sigma_{1 \mid 0}$ and $P_{1}$, the realizations of the noise processes $x_{1},\left\{w_{k}\right\}$, $\left\{v_{k}\right\},\left\{n_{k}\right\}$, and the realization of $\left\{\gamma_{k}\right\}$, the sequence of packet arrival successes or failures. Our aim is to compute and to characterize the probability cumulative distribution functions (cdfs) of these escape times as the communication link's arrival probability, $P_{\gamma}$, changes. The results of [20] establish a lower bound on $P_{\gamma}$ for the expected value of the conditional estimator covariance to be finite for all time, i.e. $\mathrm{E}\left[\Sigma_{k \mid k}\right]<\infty$, which can occur even though the estimate error itself escapes any bounded domain with probability one.

The inclusion of the output escape time problem differs from the analysis of $[20,19]$, which considers estimator behavior only, and brings us more into contact with works such as [17] which study stochastic stabilization. We note that for the state-estimate-feedback controlled system, the covariance of output $y_{k}$ depends on the covariance of the controlled state $x_{k}$, which in turn depends on the covariance of the state estimation error, $\tilde{x}_{k \mid k}$.

For specific $\gamma_{k}$ sequences, we introduce the following definition:

Definition 4 (Conditional escape time) For bounded domain $\mathcal{D}$ and stochastic process $\left\{\xi_{k}\right\}$ adapted to the sequence $\left\{\gamma_{k}\right\}$, the conditional escape time $c d f, \Phi_{e}(k)$, is the escape time cdf from $\mathcal{D}$ for a given sequence, $\left\{\gamma_{k}\right\}$, of successful and dropped communications packets.

In particular, we shall identify a special set of conditional escape times associated with unsuccessful communication from time 1 onwards.

Definition 5 (Survival time) We define the survival time cdf for $\left\{\xi_{k}\right\}, \Phi_{s}(k)$, as the conditional escape time cdf for the domain $\mathcal{D}$ with sequence $\left\{\gamma_{k}=0, k=1,2, \ldots\right\}$. 


\section{Escape Time Markov Chain Analysis}

This section provides the fundamental theoretical support for these calculations, which later will be applied to examples.

\subsection{General Markov Chain model}

Following Definition 1, given a closed domain $\mathcal{D} \subset \mathbb{R}^{d}$ and a stochastic process $\left\{\xi_{k}: k=1, \ldots\right\}$ on $\mathbb{R}^{d}$, the escape time of $\xi_{k}$ from $\mathcal{D}$ is described by a Markov chain. This is a general result concerning adapted processes and is not limited to linear Gaussian systems nor to hyperspherical domains.

Theorem 2 (Principal theoretical result [7]) For stochastic process $\left\{\xi_{k}: k=1, \ldots\right\}$ the random variable

$$
J_{k+1}= \begin{cases}1, & \text { if } \xi_{k} \in \mathcal{D} \text { and } J_{k}=1 \\ 0, & \text { otherwise, }\end{cases}
$$

is a Markov process and, denoting

$$
\Pi_{k}=\left[\begin{array}{l}
\operatorname{Pr}\left(J_{k}=1\right) \\
\operatorname{Pr}\left(J_{k}=0\right)
\end{array}\right]
$$

is described by the Markov chain.

$$
\Pi_{k+1}=\left[\begin{array}{cc}
\alpha_{k} & 0 \\
1-\alpha_{k} & 1
\end{array}\right] \Pi_{k},
$$

where

$$
\alpha_{k}=\operatorname{Pr}\left(J_{k+1} \mid J_{k}=1\right)
$$

The escape time of the stochastic process $\left\{\xi_{k}\right\}$ is the value of $k$ when $J_{k}=0$ for the first time.

Proof: From the definition (13), $J_{k}$ satisfies the Markov property.

$\operatorname{Pr}\left(J_{k+1} \mid J_{k}, \ldots J_{1}\right)=\operatorname{Pr}\left(J_{k+1} \mid J_{k}\right)$,

and so $\left\{J_{k}\right\}$ is described by a Markov chain. The transition matrix of this chain is given by

$$
\left[\begin{array}{ll}
\operatorname{Pr}\left(J_{k+1}=1 \mid J_{k}=1\right) & \operatorname{Pr}\left(J_{k+1}=1 \mid J_{k}=0\right) \\
\operatorname{Pr}\left(J_{k+1}=0 \mid J_{k}=1\right) & \operatorname{Pr}\left(J_{k+1}=0 \mid J_{k}=0\right)
\end{array}\right]
$$

The $(1,2)$-element of this matrix is zero, because there is no possibility of moving from $J_{k}=0$ to $J_{k+1}=1$.
Corollary 1 For a given $\left\{\gamma_{k}\right\}$ sequence, the corresponding $\left\{\alpha_{k}\right\}$ sequence describes the evolution of the conditional escape time $c d f$, $\Phi_{e}(k)$,

$$
\begin{aligned}
\operatorname{Pr}\left(J_{k+1}=0\right) & =\left(1-\alpha_{k}\right) \operatorname{Pr}\left(J_{k}=1\right)+\operatorname{Pr}\left(J_{k}=0\right), \\
\Phi_{e}(k+1) & =\left(1-\alpha_{k}\right)\left[1-\Phi_{e}(k)\right]+\Phi_{e}(k),
\end{aligned}
$$

or,

$$
\Phi_{e}(k+1)=\left(1-\alpha_{k}\right)+\alpha_{k} \Phi_{e}(k),
$$

whence

$$
\Phi_{e}(k)=1-\prod_{i=0}^{k-1} \alpha_{i}
$$

Proof: The final result (16) follows by rewriting (15) as

$$
\left[1-\Phi_{e}(k+1)\right]=\alpha_{k}\left[1-\Phi_{e}(k)\right],
$$

and recognizing that $1-\Phi_{e}(1)=\alpha_{0}$.

\subsection{Computation of $\alpha_{k}$ for linear systems}

As developed in Theorem 2, the escape time of stochastic process $\left\{\xi_{k}\right\}$ is governed by a Markov chain and the probability transition matrix is determined by a sole scalar value $\alpha_{k}$. For any escape time problem, $\alpha_{k}$ might be computed as

$$
\alpha_{k}=\frac{\operatorname{Pr}\left(\xi_{k+1} \in \mathcal{D}, \xi_{k} \in \mathcal{D}, \ldots, \xi_{1} \in \mathcal{D}\right)}{\operatorname{Pr}\left(\xi_{k} \in \mathcal{D}, \ldots, \xi_{1} \in \mathcal{D}\right)} .
$$

This requires the computation of a $(k+1) n$-dimensional and a $k n$-dimensional multivariate Gaussian cdf, as could in the Gaussian case in principle be carried out using mvncdf in MATLAB. However, this is problematic due to the growth of dimension of the argument. We now present an alternative recursion for the computation of $\alpha_{k}$ for linear systems.

Theorem 3 Consider the time-varying stochastic linear system

$$
\xi_{k+1}=F_{k} \xi_{k}+\omega_{k}
$$

with: $\xi_{1}$ possessing a pdf, $f_{\xi_{1}}(z)$, and $\left\{\omega_{k}\right\}$, independent from $\xi_{1}$, white and with pdfs $f_{\omega_{k}}(z)$.

Take

$$
h_{0}(z)=f_{\xi_{1}}(z),
$$

and define the sequence of (pdf) functions $\left\{h_{k}: \mathbb{R}^{n} \rightarrow \mathbb{R}_{+}\right\}$

$$
h_{k}(z)=\frac{1}{\int_{\mathcal{D}} h_{k-1}(w) d w} \int_{\mathcal{D}} f_{\omega_{k}}\left(z-F_{k} w\right) h_{k-1}(w) d w .
$$


Then

$$
\alpha_{k}=\int_{\mathcal{D}} h_{k}(w) d w
$$

Proof: This is proven in the Appendix.

Theroem 3 permits calculation of $\left\{\alpha_{k}\right\}$ for (17) for any process noise pdfs, even time-varying. In the sequel for comparison with earlier results, we shall limit investigation to either Gaussian or Gaussian-plus-uniform noise

Corollary 2 For linear Gaussian system (17) with initial state $\xi_{1} \sim N\left(0, \rho_{1}\right)$ with $\rho_{1}>0$, and $\omega_{k} \sim N\left(0, \Omega_{k}\right)$, white and independent from $\xi_{1}, \alpha_{k}$ is given by the recursion (18-20) with

$$
\begin{aligned}
& f_{\xi_{1}}(z)=\frac{1}{(2 \pi)^{n / 2}\left|\rho_{1}\right|^{1 / 2}} \exp \left(-\frac{1}{2} z^{t} \rho_{1}^{-1} z\right), \\
& f_{\omega_{k}}\left(z-F_{k} w\right)= \\
& \frac{1}{(2 \pi)^{n / 2}\left|\Omega_{k}\right|^{1 / 2}} \exp \left[-\frac{1}{2}\left(z-F_{k} w\right)^{T} \Omega_{k}^{-1}\left(z-F_{k} w\right)\right] .
\end{aligned}
$$

For the same system and initial state but with $\omega_{k}=v_{k}+n_{k}$ where $v_{k} \sim N\left(0, R_{k}\right)$ and $n_{k} \sim U\left(-\zeta / 2^{b}, \zeta / 2^{b}\right)$ with $\left\{v_{k}\right\}$ and $\left\{n_{k}\right\}$ independent and white, $\alpha_{k}$ is given the recursion (18-20) with $f_{\xi_{1}}(z)$ as above and $f_{\omega_{k}}(z)$ given by $f_{v+n}(z)$ from (5).

In both the estimator and the output escape problems, a given $\left\{\gamma_{k}\right\}$ sequence and initial state estimate covariance determine the associated Kalman gain sequence $\left\{L_{k}\right\}$, which captures the time-variability of the problem. Both cases can be subsumed into the study of the linear system (11) with the corresponding escape set. For example, the corresponding escape set of output escape time $\mathcal{D}$ and initial covariance $\mathcal{C}$ :

$$
\mathcal{D}=\left\{\left[\begin{array}{c}
x_{k} \\
\tilde{x}_{k \mid k-1} \\
y_{k}
\end{array}\right]:\left|y_{k}\right|_{\infty} \leq \zeta\right\} ; \mathcal{C}=\left[\begin{array}{c}
P_{1} \\
\Sigma_{1 \mid 0} \\
C P_{1} C^{T}+R
\end{array}\right]
$$

The escape set of estimation escape time uses $\left\{\left|\tilde{x}_{k \mid k-1}\right|_{\infty} \leq\right.$ $G\}$ instead of $\left\{\left|y_{k}\right|_{\infty} \leq \zeta\right\}$ in above equation.

For the estimator or for the output, the results of this section permit the calculation of the conditional escape time cdf for any given packet arrival sequence, $\left\{\gamma_{k}\right\}$. Since in this case, the system matrices, $F_{k}$, are known.

With $\gamma_{k}=0, k=1,2, \ldots$, the survival time analysis for both the estimator escape and for the output escape with linear systems is a time-invariant analysis, since the Kalman gain $L_{k}=0$. Accordingly, the study of survival time can be explicitly conducted, which provides insight into the processes underlying escape times, which usually require simulation for evaluation.

\subsection{Properties of output escape time for linear time-} invariant Gaussian systems

Denote by $\Phi_{e}(t)$ the cdf of the output escape time for the linear time-invariant Gaussian system

$$
\begin{aligned}
x_{k+1} & =A x_{k}+B u_{k}+w_{k}, \\
y_{k} & =C_{k}+v_{k},
\end{aligned}
$$

with

$$
\begin{gathered}
u_{k}=-K \hat{x}_{k \mid k} ; \quad x_{1} \sim N\left(0, P_{1}\right) \\
w_{k} \sim N(0, Q) ; \quad v_{k} \sim N(0, R) .
\end{gathered}
$$

By Lemma 1 since $\mathcal{D}=\left\{\left|y_{k}\right|_{\infty} \leq \zeta\right\}$ is bounded, $\Phi_{e}(t) \rightarrow$ 1 as $t \rightarrow \infty$. For the same $\gamma$-sequence (which means the same $F_{k}, \omega_{k}$ in Theorem 3), $\Phi_{e}$ is a function of $t, P_{1}, Q$, $R$ and $\zeta$. From the Theorem 3, we further have the following properties holding for all $t \geq 1$ where the superscript delineates each of two different cases:

$$
\begin{aligned}
& \left\{P_{1}^{1} \geq P_{1}^{2}\right\} \cup\left\{Q^{1} \geq Q^{2}\right\} \cup\left\{R^{1} \geq R^{2}\right\} \cup\left\{\zeta^{1} \leq \zeta^{2}\right\} \\
& \Longrightarrow \Phi_{e}\left(t, P_{1}^{1}, Q^{1}, R^{1}, \zeta^{1}\right) \geq \Phi_{e}\left(t, P_{1}^{2}, Q^{2}, R^{2}, \zeta^{2}\right),
\end{aligned}
$$

The proof of this property appears in the Appendix.

Theorem 4 For the linear time-invariant Gaussian system (22-23), the cdf of the survival time, $\Phi_{s}$, admits the following implication

$$
\begin{aligned}
& P_{1}^{1} \geq A^{n} P_{1}^{2} A^{n^{T}}+A^{n-1} Q A^{n-1^{T}}+\cdots+A Q A^{T}+Q \\
& \Longrightarrow \Phi_{s}\left(t, P_{1}^{1}, Q, R, \zeta\right) \geq \Phi_{s}\left(t+n, P_{1}^{2}, Q, R, \zeta\right) .
\end{aligned}
$$

Theorem 4 will be used when studying the effect of quantization errors on the escape time.

\section{Escape time computational examples}

\subsection{Example 1: Estimator survival time}

Recall that the survival time is the escape time when no data packets are received at times $k=1,2, \ldots$. Because Theorem 3 provides an explicit formula for $\alpha_{k}$, we study first the survival time of the estimator. This example, while simple, permits revealing comparisons between the escape/survival time analysis and the moment-based methods of $[20,19]$. 
Consider the system

$$
\begin{aligned}
& A=1.5, B=1, C=1, Q=0.005, R=0.001, \zeta=10, \\
& \text { with } \Sigma_{1 \mid 0}=A R_{\mathrm{eff}} A^{T}+Q=0.00725,
\end{aligned}
$$

which corresponds to the largest possible conditional prediction covariance immediately after receiving a single data sample. We compute the survival time cdf using Theorems 2 and 3 which is a time-invariant system transient analysis yielding explicitly the survival cdf. The probability density function is plotted in Figure 4.

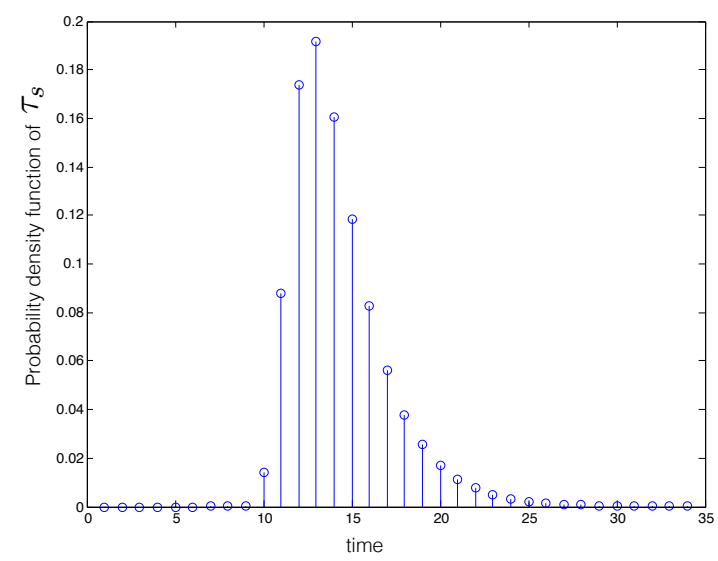

Fig. 4. Probability density function of estimator survival time for Example 1.

The calculation of the density function of $\tau_{s}$ was verified, needlessly, by simulation. Figure 5 displays three sample $\tilde{x}_{k+1 \mid k}$ trajectories of differing survival times: 8,12 , and 19, from a Monte Carlo simulation with initial covariance $\Sigma_{1 \mid 0}=0.00725$. We have selected three trajectories which escaped with positive state-estimate error values. From the

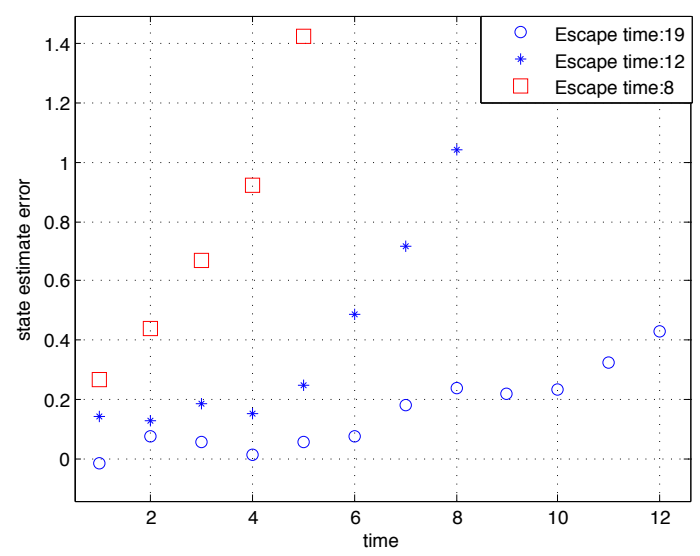

Fig. 5. Three different estimator survival time trajectory samples for Example 1 with initial error covariance $\Sigma_{1 \mid 0}=0.00725$. problem description, the state-estimate error satisfies,

$$
\tilde{x}_{k+1 \mid k}=(1.5)^{k} \tilde{x}_{1 \mid 0}+\sum_{j=1}^{k}(1.5)^{k-j} w_{j},
$$

which displays the relative importance of the random terms. Since $w_{k} \sim N(0,0.005)$, once the state exceeds roughly 0.2 (three $\sigma$ ) in magnitude it becomes most unlikely that $w_{k}$ will arise to bring the error back to a small magnitude. Once the three trajectories exceed this value, we see that they all escape with roughly similar behavior, i.e. at a rate of $1.5^{k}$. The difference between the trajectories lies in their residence time in the neighborhood $|\tilde{x}|<0.2$. Here the disturbance process is as likely to drag the error back towards small magnitudes as it is to increase the magnitude, resulting in some trajectories remaining close to zero for an extended time. This is captured by the Markov analysis.

\subsection{Markov versus covariance comparison}

Comparing the current Markov results to the covariance calculations of [20], we see that, in both survival time analyses, the conditional covariance is given by

$$
\Sigma_{k+1 \mid k}=A^{k} \Sigma_{1 \mid 0} A^{k^{T}}+\sum_{j=0}^{k-1} A^{j} Q A^{j^{T}} .
$$

In contrast to the Markov analysis, covariance $\Sigma_{k+1 \mid k}$ may be used to compute directly the probabilities $\operatorname{Pr}\left(\left|\tilde{x}_{k+1 \mid k}\right|_{2} \leq\right.$ $\zeta)$ and $\operatorname{Pr}\left(\left|\tilde{x}_{k+1 \mid k}\right|_{2}>\zeta\right)$ using the normal cdf. Whence, the escape probability may be approximated via

$\operatorname{Pr}\left(\left|\tilde{x}_{k+1 \mid k}\right|_{\infty}>\zeta\right) \times \prod_{j=1}^{k} \operatorname{Pr}\left(\left|\tilde{x}_{k+1-j \mid k-j}\right|_{\infty}<\zeta\right)$.

This calculation may be performed using the stationary expected conditional covariance or using the data-dependent covariance, $\Sigma_{k+1 \mid k}$, derived from the $\left\{\gamma_{k}\right\}$ sequence.

Figure 6 displays three curves computed for Sinopoli's example from [20] in the region where the expected conditional covariance is finite, i.e. $P_{\gamma}>0.36$. Here,

$$
\begin{aligned}
& A=\left[\begin{array}{cc}
1.25 & 0 \\
1 & 1.1
\end{array}\right], Q=\left[\begin{array}{cc}
20 & 0 \\
0 & 20
\end{array}\right], P_{\gamma}=0.4 \\
& \bar{M}=\left[\begin{array}{ll}
67.36 & -.052 \\
-.052 & 22.73
\end{array}\right], C=\left[\begin{array}{ll}
1 & 1
\end{array}\right], R=2.5, \\
& \zeta=100 .
\end{aligned}
$$




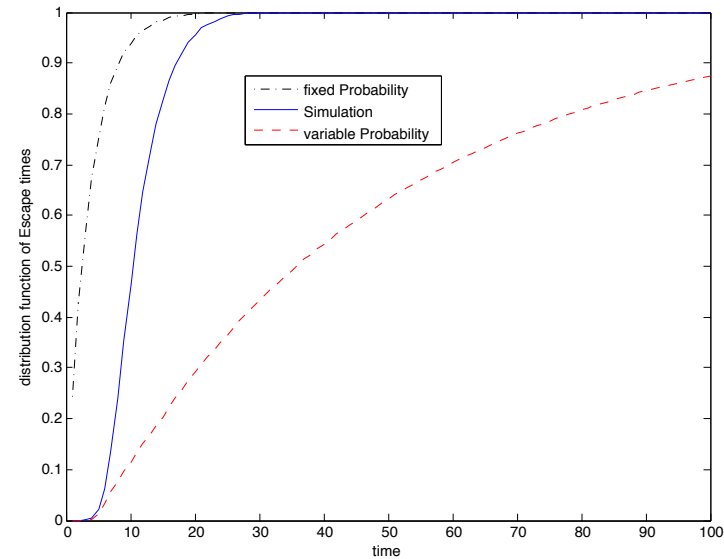

Fig. 6. Comparison of escape time cumulative distribution functions for Sinopoli's Example: unbroken curve - simulation, dashdot curve - estimate using fixed expected conditional covariance and (25), dashed curve - estimate using time-varying conditional covariance and (25) then averaged over 1000 trials.

This calculation demonstrates that attention solely to the covariance is problematic and, further, that the stationary expected conditional covariance is pessimistic in its implication regarding escape time, while the time-varying covariance is overly optimistic. This corroborates the results of [19], where the expected value of the conditional covariance is replaced by analysis of its probabilistic behavior.

\subsection{Example 1 redux: Output survival times with quantized data}

We return to the scalar-state Example 1, but now with stateestimate feedback control. The parameters of the controlled system and output signal bound, $\zeta$, are:

$$
\begin{aligned}
& A=1.5, B=1, C=1, D=0, K=1.2, \\
& Q=0.005, R=0.001, \zeta=10 .
\end{aligned}
$$

We consider two quantization scenarios; 5-bit quantization of $y_{k}$, and 16-bit quantization.

Figure 7 depicts the cdfs for 16-bit (blue) and 5-bit (red) for $P_{\gamma}$ taking values in $\{0.2,0.3,0.4,0.5\}$. It is noteworthy that the explicitly quantified difference in survival times of the differing bitrates, described by Theorem 4 in the case where $P_{\gamma}=0$, is exacerbated when $P_{\gamma}$ increases.

Figure 8 shows the variation in escape times for increasing values of the bound, $\zeta$, defining the escape domain $\mathcal{D}$, and for fixed $P_{\gamma}=0.2$. The bound $\zeta$ takes values in the set $[1,10,100,500]$. This affects the quantization intervals and, in turn, affects: the effective measurement noise, $R_{\text {eff }}$, as in (6); the estimator covariance value $\Sigma_{k+1 \mid k}$; and the initial controlled state covariance, $P_{1}$.

It is apparent that, for small numbers of bits, the quantization error becomes a significant factor when its effect on $P_{1}$

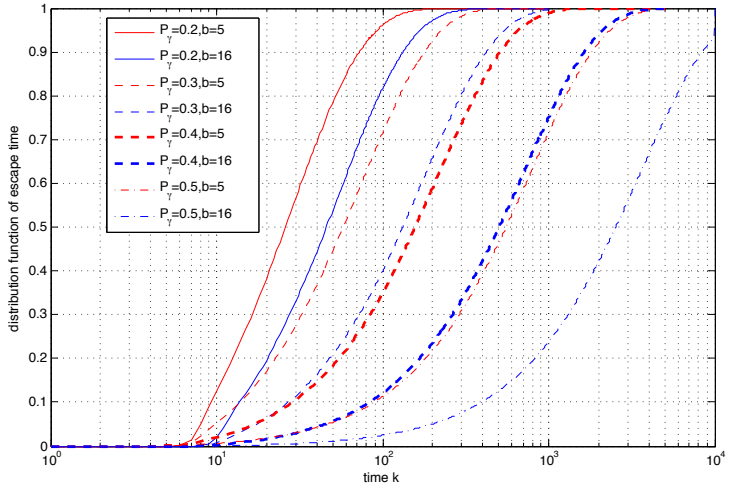

Fig. 7. Semilog plot of cumulative distribution functions of output escape times for 5-bit (red) and 16-bit (blue) quantized measurements as the probability of successful packet arrival, $P_{\gamma}$, varies. The order of the plots left-to-right in the center corresponds to the order down the legend.

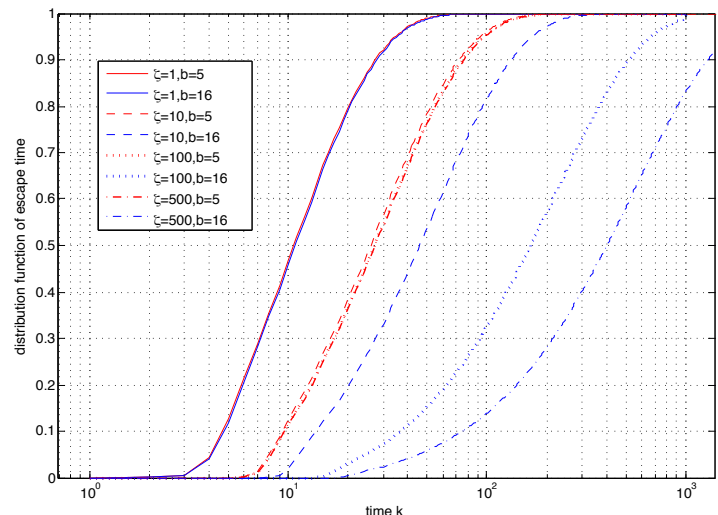

Fig. 8. Cumulative distribution functions of controlled output escape times for Example 1 with $\zeta=1,10,100,500$, and with $P_{\gamma}=0.2$. Results are shown for 5-bit quantization (red) and 16-bit quantization (blue).

dominates that of the process noise $Q$. When the initial state covariance $P_{1}$ becomes large enough, the dependence of escape time on $\zeta$ depends primarily on growth in $\zeta$ versus the maximal eigenvalue of $A$. Thus, for these $\zeta$ values and 5-bit quantization, we see little apparent variation in the escape time statistics past $\zeta=10$, while the 16-bit quantization escape times continue to grow dramatically with $\zeta$.

\section{Bitrate assignment and escape time}

Communications issues of intermittency and limited bitrate may be combined for joint analysis and design, particularly in the realm of partitioning the available bitrate as a hedge against the deleterious effects of dropped packets. Evidently this is a rudimentary coding question, since one can sacrifice some bits to accommodate error recovery. This represents a departure from $[20,19]$, whose analysis is restricted to intermittency alone. Although, [19] countenances retransmission of data within each packet, albeit at no cost to the 
communication rate. More recently, [13] has studied the application of redundant parallel channels in the amelioration of packet loss effects, again without addressing quantization issues arising due to limited bitrates.

Sinopoli et al. [20] assume that the measurement matrix $C=I$ in (2) while [19] assumes that each packet contains $r$ successive measurements, where $r$ is the the least value for which

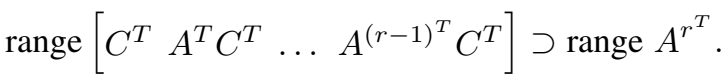

Further, these data packets are not bitrate limited. In either case, the arrival of any single packet suffices for reconstruction of the state estimate with conditional covariance less than a specific bounded quantity derived from the Information Filter, see [1].

With a limited bitrate and $r>1$, the assignment of bits within a measurement packet to retransmitted data comes at a cost in terms of the state estimate error, because multiply arriving copies of data carry no information and the associated bits might better have been used for the new data. We next analyze by simulation the effect of such bitrate assignment on escape time.

Suppose that the bits at any time are assigned to the most recent data and also to some fraction of the earlier data, as is illustrated in Figure 9. This redundancy in data transmis-

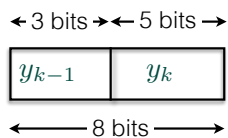

Fig. 9. Bit allocation scheme. Here 3 of 8 bits at time $k$ are assigned to $y_{k-1}$ and 5 bits to $y_{k}$.

sion should provide some protection from packet loss at the expense of estimation accuracy. This impact can be subtle, since the reduction in bits for $y_{k}$ affects both the limiting filtered estimate and controlled state covariances.

Our example takes

$$
\begin{aligned}
& A=\left[\begin{array}{cc}
0 & 1.5 \\
1.5 & 0
\end{array}\right], B=\left[\begin{array}{l}
1 \\
0
\end{array}\right], C=\left[\begin{array}{ll}
1 & 0
\end{array}\right], D=0, \\
& Q=\left[\begin{array}{cc}
0.05 & 0 \\
0 & 0.05
\end{array}\right], R=0.01, \zeta=5, K=[-1.1,1.66] .
\end{aligned}
$$

Case A: We take 8 bits for $y_{k}$ at each time $k$. Then the appropriate initial covariances are

$$
\Sigma_{0 \mid 0}=\left[\begin{array}{cc}
0.010 & 0 \\
0 & 0.0073
\end{array}\right] ; P_{0}=\left[\begin{array}{cc}
1.027 & 1.353 \\
1.353 & 2.361
\end{array}\right]
$$

Case B: We take 4 bits each for $y_{k-1}$ and for $y_{k}$ with corresponding

$$
\Sigma_{0 \mid 0}=\left[\begin{array}{cc}
0.075 & 0 \\
0 & 0.219
\end{array}\right], P_{0}=\left[\begin{array}{ll}
2.404 & 3.099 \\
3.099 & 5.459
\end{array}\right] .
$$

Escape time cdfs are shown in Figure 5 for the case where $P_{\gamma}=0.3$. We see that, in this case, the retransmission strategy serves to extend the time for escape attributable to nefarious sequences $\left\{\gamma_{k}\right\}$ at the cost of increasing the likelihood of early escape ascribed to the concomitant increase in initial covariances.

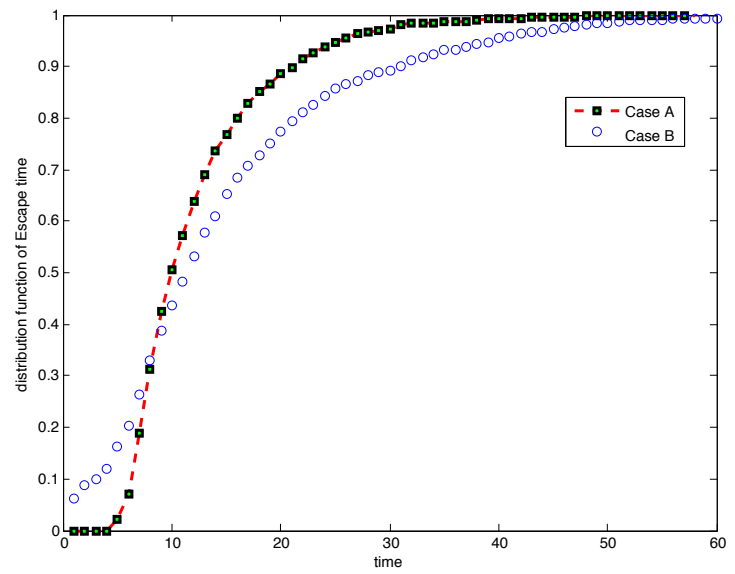

Fig. 10. Output escape time cdfs with single transmission (Case A) and split bitrate signal retransmission (Case B) when $P_{\gamma}=0.3$

\section{Comments and conclusions}

We have presented an approach to the study of state estimation and state-estimate feedback stabilization with intermittent and quantized measurements. This is based on escape time analysis and computation, which is compared to earlier works in estimation and stabilization with differing descriptions of the properties - finite expected conditional covariance and mean-square stabilization - and of the communication system - intermittent but exact and quantized but certain. The central result is that the escape time can be described by a Markov chain, which in the linear case is easily computed. This yields much more precise evaluation of behavior than covariance calculations, even in the Gaussian case. Our analysis has included the treatment of quantization effects in output escape time problems and shown that bitrate choice can have a profound effect on the output escape time properties of a controlled system.

\section{Coda \& Acknowledgement}

The authors are grateful to the perspicacious anonymous reviewers who pointed out important technical errors in early 
versions of this paper. The thrust of these problems was to replicate in the escape time framework the resetting of covariance-based analyses once certain sequences of successful packets arrive. In the Kalman filtering context, this is used to guarantee the return to an upper bounded conditional covariance matrix - the actual bound follows from the Information Filter formulation of the problem. This resetting permits the separation of the analysis of future behavior from that prior to the reset. In the escape time formulation this separation is no longer possible and it is instructive to understand why.

In the Gaussian case, one (including the authors) might be expected to use the following relation following from (14).

$$
\begin{aligned}
\alpha_{k} & =\operatorname{Pr}\left(J_{k+1}=1 \mid J_{k}=1\right), \\
& =\operatorname{Pr}\left(J_{k+1}=1 \mid J_{k}=1, J_{k-1}=1\right), \\
& =\frac{\operatorname{Pr}\left(J_{k+1}=1, J_{k}=1 \mid J_{k-1}=1\right)}{\operatorname{Pr}\left(J_{k}=1 \mid J_{k-1}=1\right)} .
\end{aligned}
$$

This latter quantity is familiar from Kalman filtering and computations such as

$$
\operatorname{Pr}\left(x_{k+1} \mid y_{k}, y_{k-1}, \ldots, y_{1}\right)=\frac{\operatorname{Pr}\left(x_{k+1}, y_{k} \mid y_{k-1}, \ldots, y_{1}\right)}{\operatorname{Pr}\left(y_{k} \mid y_{k-1}, \ldots, y_{1}\right)}
$$

However for escape times, the conditioning is not on the specific value taken by a signal such as $y_{k}$. Instead, the conditioning is over the residence of $x_{k} \in \mathcal{D}$ and the probabilities above need to be computed as integrals over all of $\mathcal{D}$. The finite-dimensionality of the Kalman filter is lost since, even though $\left(x_{k+1}, x_{k}\right)$ are jointly Gaussian, the conditional probability $\operatorname{Pr}\left(x_{k+1} \mid x_{k} \in \mathcal{D}\right)$ is not Gaussian. This is evident in the precise calculations of Theorem 3.

\section{References}

[1] B.D.O. Anderson and J.B. Moore. Optimal Filtering. Prentice-Hall, Englewood Cliffs, New Jersey, 1979.

[2] D.F. Delchamps. Stabilizing a linear system with quantized state feedback. IEEE Transactions on Automatic Control, 35(8):916 -924, August 1990.

[3] J. Evans and V. Krishnamurthy. Optimal sensor scheduling for Hidden Markov Models. In Proceedings of the 1998 IEEE International Conference on Acoustics, Speech and Signal Processing, 1998, volume 4, pages 2161 -2164, May 1998.

[4] R. Evans, V. Krishnamurthy, G. Nair, and L. Sciacca. Networked sensor management and data rate control for tracking maneuvering targets. IEEE Transactions on Signal Processing, 53(6): 1979 - 1991, June 2005.

[5] M.I. Freidlin and A.D. Wentzell. Random perturbations of dynamical systems. Springer Verlag, Berlin, 1998.

[6] J.A. Fulton, R.R. Bitmead, and R.C. Williamson. Smoothing approaches to reconstruction of missing data in array processing. In D. Cochran, W. Moran, and L.B. White, editors, Defence Applications of Signal Processing, pages 87-94. Elsevier, 2001.
[7] C.C. Huang and R.R. Bitmead. Escape-time formulation of state estimation and stabilization with intermittent communication. In European Control Conference, pages 99-104, Zürich, 2013.

[8] M. Huang and S. Dey. Stability of Kalman filtering with Markovian packet losses. Automatica, 43(4):598-607, 2007.

[9] S. Kar, B. Sinopoli, and J.M.F. Moura. Kalman filtering with intermittent observations: Weak convergence to a stationary distribution. IEEE Transactions on Automatic Control, 57(2):405 -420, February 2012.

[10] R.Z. Khasminskii. Stochastic stability of differential equations. Sijthoff \& Noordhoff, Alphen aan den Rijn, 1980.

[11] R.J.A. Little and D.B. Rubin. Statistical Analysis with Missing Data. John Wiley \& Sons, New York, 1987.

[12] S.M. Meerkov and T. Runolfsson. Residence time control. IEEE Transactions on Automatic Control, 33(4):323 -332, April 1988.

[13] A.R. Mesquita, J.P. Hespanha, and G.N. Nair. Redundant data transmission in control/estimation over lossy networks. Automatica, 48:1612-1620, 2012.

[14] P. Minero, L. Coviello, and M. Franceschetti. Stabilization over Markov feedback channels: The general case. IEEE Transactions on Automatic Control, 58(2):349-362, February 2013.

[15] P. Minero, M. Franceschetti, S. Dey, and G. N. Nair. Data rate theorem for stabilization over time-varying feedback channels. IEEE Transactions on Automatic Control, 54(2):243255, February 2009.

[16] Yilin Mo and B. Sinopoli. Kalman filtering with intermittent observations: Tail distribution and critical value. IEEE Transactions on Automatic Control, 57(3):677 -689, March 2012.

[17] G. Nair and R.J. Evans. Stabilizability of stochastic linear systems with finite feedback data rates. SIAM Journal on Control and Optimization, 43(2):413-436, 2004.

[18] F.C. Schweppe and E.J. Handschin. Static state estimation in electric power systems. Proceedings of the IEEE, 62(7):972 - 982, July 1974.

[19] Ling Shi, M. Epstein, and R.M. Murray. Kalman filtering over a packet-dropping network: a probabilistic perspective. IEEE Transactions on Automatic Control, 55(3):594-604, 2010.

[20] B. Sinopoli, L. Schenato, M. Francheschetti, K. Poolla, M.I. Jordan, and S.S. Sastry. Kalman Filtering with intermittent observations. IEEE Transactions on Automatic Control, 49(9):1453-1464, September 2004.

[21] D.N. Swingler and R.S. Walker. Line-array beamforming using linear prediction for aperture interpolation and extrapolation. IEEE Transactions on Acoustics, Speech and Signal Processing, 37(1):16 -30, January 1989.

[22] S. Tatikonda and S. Mitter. Control over noisy channels. IEEE Transactions on Automatic Control, 49(7):1196 - 1201, July 2004.

[23] S. Tatikonda and S. Mitter. Control under communication constraints. IEEE Transactions on Automatic Control, 49(7):1056 - 1068, July 2004.

[24] S.R.S. Varadhan. Large deviations and applications. SIAM, Philadelphia, 1984.

[25] B. Widrow and I. Kollar. Quantization Noise Roundoff Error in Digital Computation, Signal Processing, Control, and Communications. Cambridge Univ. Press, 2008.

[26] N. Wiener. The Extrapolation, Interpolation and Smoothing of Stationary Time Series with Engineering Applications. Wiley, New York, 1949. 
[27] D. Williamson. Finite wordlength design of digital Kalman filters for state estimation. IEEE Transactions on Automatic Control, 30(10):930 - 939, October 1985.

[28] D. Williamson and K. Kadiman. Optimal finite wordlength linear quadratic regulation. IEEE Transactions on Automatic Control, 34(12):1218 -1228, December 1989.

[29] Wing Shing Wong and R.W. Brockett. Systems with finite communication bandwidth constraints. I. State estimation problems. IEEE Transactions on Automatic Control, 42(9):1294 -1299, September 1997.

[30] Wing Shing Wong and R.W. Brockett. Systems with finite communication bandwidth constraints. II. Stabilization with limited information feedback. IEEE Transactions on Automatic Control, 44(5):1049 -1053, May 1999.

[31] Keyou You and Lihua Xie. Minimum data rate for mean square stabilizability of linear systems with Markovian packet losses. IEEE Transactions on Automatic Control, 56(4):772 - 785, April 2011.

[32] J. Zabzcyk. Exit problems and control theory. Systems \& Control Letters, 6:165-172, 1985.

\section{Appendix}

\subsection{Proof of Lemma 1}

Without loss of generality take $\mathcal{D}$ to be a hypersphere in $\mathbb{R}^{n}$, i.e. $|x|^{2} \leq r$ for some $r>0$. If this is not the case then, since $\mathcal{D}$ is compact, we can replace $\mathcal{D}$ by another compact set $\mathcal{D}^{+}$ which is a hypersphere containing $\mathcal{D}$ and perform the same analysis. Escape from $\mathcal{D}^{+}$certainly implies escape from $\mathcal{D}$.

At time $k$ with state $x_{k}$ and control $u_{k}$, both of which are independent from $w_{k}$ under the conditions of the lemma, the condition for $x_{k+1}$ to lie outside $\mathcal{D}$ is that

$$
A x_{k}+B u_{k}+w_{k} \in \mathcal{D}^{c}
$$

the complement of $\mathcal{D}$. Since $\mathcal{D}$ is a hypersphere centered at the origin and $w_{k}$ is zero mean Gaussian

$$
\operatorname{Pr}\left(w_{k}+v \in \mathcal{D}\right) \leq \operatorname{Pr}\left(w_{k} \in \mathcal{D}\right)=\beta<1,
$$

for any vector $v$. This argument can be extended to multiple steps as follows.

$$
\begin{aligned}
& \operatorname{Pr}\left(w_{k}+v_{k} \in \mathcal{D}, w_{k+1}+v_{k+1} \in \mathcal{D}\right) \\
& =\operatorname{Pr}\left(w_{k+1}+v_{k+1} \in \mathcal{D} \mid w_{k}+v_{k} \in \mathcal{D}\right) \operatorname{Pr}\left(w_{k}+v_{k} \in \mathcal{D}\right), \\
& \leq \beta \operatorname{Pr}\left(w_{k}+v_{k} \in \mathcal{D}\right) \\
& \leq \beta^{2}
\end{aligned}
$$

So the probability of escape by time $k$ is bounded by $\beta^{k}$ and, by the Borel-Cantelli Lemma, we have the probability of no escape is zero.

\subsection{Proof of Theorem 3}

The proof hinges on the careful application of the Markov property. Denote by $p$ the general pdf where the argument specifies the pdf and define

$$
\begin{aligned}
h_{k}\left(\xi_{k+1}\right) & =p\left(\xi_{k+1} \mid J_{k}=1\right), \\
& =\frac{p\left(\xi_{k+1}, J_{k}=1\right)}{\operatorname{Pr}\left(J_{k}=1\right)} .
\end{aligned}
$$

Now note that the numerator

$$
\begin{aligned}
& p\left(\xi_{k+1}, J_{k}=1\right) \\
& =\int_{\xi_{k} \in \mathcal{D}} p\left(\xi_{k+1} \mid \xi_{k}, J_{k-1}=1\right) p\left(\xi_{k}, J_{k-1}=1\right) d \xi_{k} \\
& =\int_{\xi_{k} \in \mathcal{D}} p\left(\xi_{k+1} \mid \xi_{k}\right) p\left(\xi_{k}, J_{k-1}=1\right) d \xi_{k} \\
& =\operatorname{Pr}\left(J_{k-1}=1\right) \int_{\xi_{k} \in \mathcal{D}} p\left(\xi_{k+1} \mid \xi_{k}\right) p\left(\xi_{k} \mid J_{k-1}=1\right) d \xi_{k} \\
& =\operatorname{Pr}\left(J_{k-1}=1\right) \int_{\xi_{k} \in \mathcal{D}} f_{\omega_{k}}\left(\xi_{k+1}-F_{k} \xi_{k}\right) h_{k-1}\left(\xi_{k}\right) d \xi_{k}
\end{aligned}
$$

where $f_{\omega_{k}}$ is the probability density function of $\omega_{k}$. [The Markov property is invoked in replacing $p\left(\xi_{k+1} \mid \xi_{k}, J_{k-1}=\right.$ 1) by $p\left(\xi_{k+1} \mid \xi_{k}\right)$.] Substituting (28) into (27) yields

$$
\begin{aligned}
& h_{k}\left(\xi_{k+1}\right) \\
& =\frac{\operatorname{Pr}\left(J_{k-1}=1\right)}{\operatorname{Pr}\left(J_{k}=1\right)} \int_{\xi_{k} \in \mathcal{D}} f_{\omega_{k}}\left(\xi_{k+1}-F_{k} \xi_{k}\right) h_{k-1}\left(\xi_{k}\right) d \xi_{k}, \\
& =\frac{1}{\alpha_{k-1}} \int_{\xi_{k} \in \mathcal{D}} f_{\omega_{k}}\left(\xi_{k+1}-F_{k} \xi_{k}\right) h_{k-1}\left(\xi_{k}\right) d \xi_{k} .
\end{aligned}
$$

Finally, integrating (26) yields

$$
\begin{aligned}
\alpha_{k} & =\operatorname{Pr}\left(\xi_{k+1} \in \mathcal{D} \mid J_{k}=1\right), \\
& =\int_{\xi_{k+1} \in \mathcal{D}} p\left(\xi_{k+1} \mid J_{k}=1\right) d \xi_{k+1}, \\
& =\int_{\xi_{k+1} \in \mathcal{D}} h_{k}\left(\xi_{k+1}\right) \mathrm{d} \xi_{k+1} .
\end{aligned}
$$

\subsection{Proof of the property (24)}

Consider the time-varying, linear, Gaussian system (11) derived from (22-23) and written in the form of (17) with zeromean initial state. That is,

$$
\xi_{k+1}=F_{k} \xi_{k}+\omega_{k}
$$


with $\xi_{0} \sim \mathcal{N}(0, \mathcal{P})$ and $\omega_{k} \sim \mathcal{N}\left(0, \Upsilon_{k}\right)$. Further, consider escape from the region $\mathcal{D}=\left\{\left|D \xi_{k}\right| \leq g\right\}$ for some constant matrix $D$. At time $\mathrm{t}$, escape of this system coincides with

$$
\Xi_{t}=\left[\begin{array}{c}
\xi_{0} \\
\xi_{1} \\
\vdots \\
\xi_{t}
\end{array}\right] \notin \mathcal{D}^{t+1}=\overbrace{\mathcal{D} \times \mathcal{D} \times \cdots \times \mathcal{D}}^{t+1 \text { times }}
$$

and $\Xi_{t}$ is Gaussian and zero mean. Now using increase to mean does not decrease, if either $\operatorname{cov}\left(\xi_{0}\right)$ increases or $\operatorname{cov}\left(\omega_{k}\right)$ increases then the covariance of $\xi_{t}$ increases and escape from the set $\mathcal{D}^{t}$ as defined above increases. By the same token, if the parameter $g$ decreases then the escape probability increases. This proves (24). 